\title{
Endoscopy by nonphysicians
}

\author{
Elaine R Gossman MD
}

$\mathrm{I}_{\mathrm{p}}^{\mathrm{n}}$

n 1945, Henry J Kaiser organized a group of physicians to provide health care for shipyard workers and their families. His foundation, Kaiser Permanente (USA), has developed into a prepaid health plan facility serving 8.4 million members in nine states and in Washington, DC, within the United States. It aspires to provide high quality, affordable, integrated health care within an organized, efficient system. A focus on preventive care in a cost-effective manner is a high priority. The Kaiser Permanente northwest region (covering northwest Oregon and southwest Washington) serves nearly one-half of a million members. Our region has utilized nurse practitioners (NPs) and physician assistants (PAs) (allied health care providers) in a variety of capacities for many years. The present paper reviews the background and current status of their role in endoscopy.

Flexible sigmoidoscopy clinics, in three locations within the region, allow convenient access for members as well as for consolidation

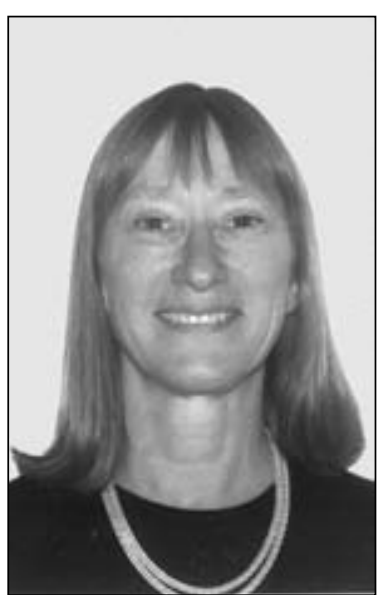

Dr Elaine R Gossman procedure time, depth of insertion of the endoscope and patient satisfaction. With proper training, continued clinical experience and supervision, an allied health clinician is generally able to provide competent care in this area.

PA education, in one of 130 accredited programs in the United States, occurs in a medical model designed to complement physician training. Classroom and laboratory instruction in medical and behavioural sciences is followed by clinical rotations in a variety of areas. PAs are licensed by each state to practice under the supervision of a physician.

Our region has been fortunate to employ a PA since 1972 in the departments of surgery and gastroenterology. His career has evolved from performing rigid sigmoidoscopy, to assisting in colonoscopy and upper endoscopy, to performing these procedures with indirect supervision. His training included a gastroenterology assistant training program for one year at the University of Washington (USA) in 1975, then of resources. The clinics have been staffed by PAs and NPs, with close interactions between gastroenterology and surgery departments since 1978. Patients are referred for screening sigmoidoscopies, as well as evaluation and management of a variety of colorectal problems. Currently, allied health care providers are trained by experienced clinicians. Didactic sessions focus on indications for the procedure, anatomy and endoscopic appearance of the anorectum and distal colon, as well as pathophysiology of common colorectal disorders. Principles of disinfection and the importance of a thorough, safely performed examination are emphasized. The trainees observe several procedures before beginning their hands-on experience. Stepwise training begins with manipulation of the shaft of the sigmoidoscope, then proceeds to experience in controlling the dials, initially withdrawing and then advancing the instrument. Training includes the use of the biopsy forceps. Certification of competence after 100 to 200 procedures is at the discretion of the supervising clinician. The American Society for Gastrointestinal Endoscopy has recommended a minimum of 25 flexible sigmoidoscopies before competence has been assessed (1). Our region does not believe that this is adequate experience for a clinician to perform the procedure without direct supervision.

Several studies (2-4) have compared examinations performed by physicians with those performed by nurse endoscopists. They uniformly report comparable quality, including complication rates, identification of polyps, mean additional training at the Portland VA Medical Center (USA) in 1978 to learn flexible sigmoidoscopy. After over 50 directly supervised flexible sigmoidoscopies, he was approved by the state board of medical examiners to perform this examination with indirect supervision. Over the next 19 years, he staffed the sigmoidoscopy clinic and continued to work closely with gastroenterologists and surgeons.

His interests led to an apprenticeship in gastroenterology for advanced procedures, including colonoscopy (5). Over a four-year period of training, with several members of the department of gastroenterology, he completed over 500 directly supervised colonoscopies leading to his approval by the Oregon Board of Medical Examiners to perform this procedure with indirect supervision. He was later approved to perform upper endoscopy after documenting over 200 procedures with supervision. In his role as a PA, it was agreed that his training include basic knowledge in the practice of clinical gastroenterology rather than have him function as a technician or proceduralist alone. Continuing education in pathology, basic physiology, pharmacology and participation in quality assurance are crucial aspects of his practice.

He currently performs approximately 1200 procedures a year. His training included the technical aspects of the procedures and cognitive skill training in identifying and interpreting the examination and managing the clinical problems. He is adept at snare polypectomy, endoscopic injections for tattooing lesions and management of gastrointestinal bleeding, 
esophageal dilation with through-the-scope balloons and over-the-wire dilators, percutaneous endoscopic gastrostomy placement and esophageal variceal banding. He is certified in procedural sedation. His skills evolved over the years with involvement in diverse inpatient and outpatient clinical scenarios. The volume of procedures he performs and his continuing medical education, through the same channels as the rest of the department, has assured his continued competence.

With the national promotion of colon cancer screening over the past several years, the demand for gastroenterologists in the United States has far exceeded the supply. Training programs were reduced in the mid-1990s, and are not expanding to meet this demand. Indications for colonoscopy continue to expand. Management of chronic hepatitis B and C viruses, growing numbers of liver transplant patients, increased membership in our region and increased member expectations have been other factors that have strained our capacity to meet the health care needs of the Kaiser members in our region. Because of the successful training of the PA to perform colonoscopy and upper endoscopy, the gastroenterology department uniformly supported the addition of another PA to the department in an effort to meet the demands for our services, while maintaining a high standard of care. In 2001, a PA who was skilled at flexible sigmoidoscopy was trained in colonoscopy and upper endoscopy. Incorporating detailed knowledge in common gastrointestinal disorders while training in the technical aspects of the procedures was emphasized. Accurate identification and interpretation of endoscopic findings, as well as the knowledge of how to manage the patients, are crucial skills. After one year of intensive education and clinical experience, he also received state board approval for these procedures.

His role extends well beyond that of colon cancer screening. Given the growing demand for gastroenterology services, in many areas beyond colon cancer screening, our department continues to be challenged in meeting the volume of referrals. The efficiencies gained by educating him in many aspects of the specialty are numerous. It is standard in our region to perform both colonoscopy and upper endoscopy at the same visit if both procedures are indicated as a way to best utilize our resources, as well as for the convenience of the patients. For example, in the evaluation of iron deficiency anemia, if the cause is not clearly determined by the colonoscopic findings, upper endoscopy with small bowel biopsy to screen for celiac sprue will be carried out at the same visit. If a patient referred for screening colonoscopy also has dysphagia, both procedures will be performed at the initial visit. His ability to manage many common gastrointestinal disorders has been a major benefit to the department.

It is not clear that our experience with PAs performing colonoscopy and upper endoscopy can be transferred to the fee-for-service sector. The prevalence of this practice in the
United States is unknown, but thought to be quite rare. Credentialing by hospitals or outpatient endoscopy centres would present barriers in most sites. Our own facilities have credentialed our PAs to perform these procedures. We require a physician in the immediate vicinity who is available to assist or advise when needed. Non-Kaiser facilities have not allowed us to utilize PAs in this capacity. Reimbursement issues by other health insurers would be significant issues. Medicare reimbursement regulations for PA services has afforded some limitations in our system. The investment of a large amount of physician time in training an allied clinician is a barrier to this expanded role. Limiting the role of the allied clinician to solely screening colonoscopies still requires a significant investment. Polypectomy skills are mandatory for screening. Injection of bleeding polypectomy sites and tattooing lesions follow as requirements. Identification of other pathology, such as inflammatory or other neoplastic conditions is important, as well as the knowledge base to deal with the findings. Recognition and appropriate management of procedural complications are inherent requirements to the performance of these procedures. The additional training for competence in upper endoscopy as a way to offer more comprehensive care in a single visit appears reasonable.

Clearly, there is a role for allied clinicians to perform flexible sigmoidoscopy primarily for colon cancer screening because the additional technical skills beyond cold forceps biopsy are not required. If a polyp is documented, complete colonoscopy to evaluate the rest of the colon is advised. Snare polypectomy is performed at the time of the colonoscopy. However, even in this setting, it is important for the sigmoidoscopist to be able to identify other pathology and have the basic knowledge to manage some problems, or to refer patients when appropriate. The clinician must understand appropriate indications and contraindications to the procedure that they are performing. They must be well trained to perform the procedure safely, thoroughly and with clinical outcomes comparable with physicians who perform the procedure. Acknowledgement of the limitations of the procedure, as well as when referral for further care is required, are crucial to the success of such a program.

\section{REFERENCES}

1. The American Society for Gastrointestinal Endoscopy. Training guidelines. <http://www.asge.org/nspages/practice/patientcare/ training/training.cfm $>$ (Version current at December 7, 2006).

2. Schoenfeld P, Lipscomb S, Crook J, et al. Accuracy of polyp detection by gastroenterologists and nurse endocopists during flexible sigmoidoscopy: A randomized trial. Gastroenterology 1999:117:312-8.

3. Maule WF. Screening for colorectal cancer by nurse endoscopists. N Engl J Med 1994;330:183-7.

4. DiSario JA, Sanowski RA. Sigmoidoscopy training for nurses and resident physicians. Gastrointest Endosc 1993;39:29-32.

5. Shneidman R, Fossati D. Use of physician assistant in gastroenterology in a community setting. Am J Gastroenterol 2000;95:AB6207. (Abst) 


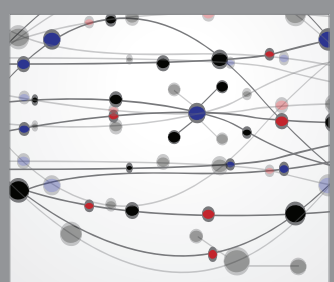

The Scientific World Journal
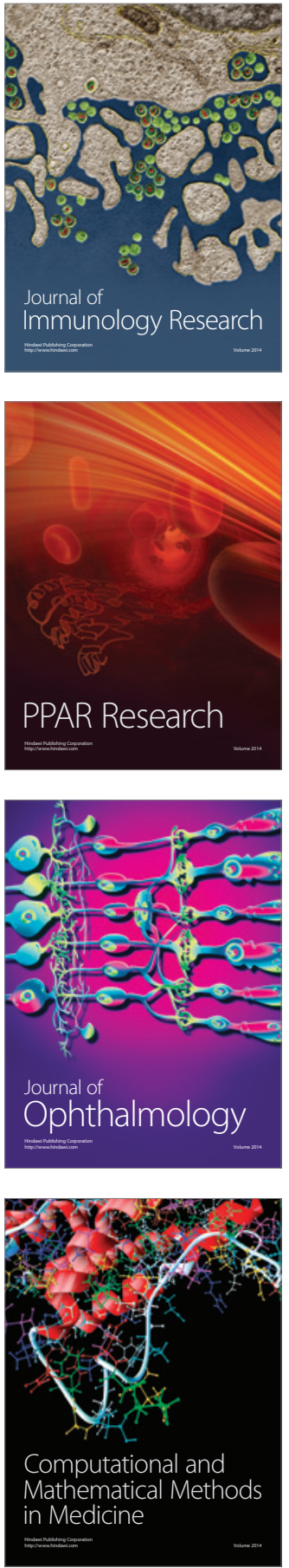

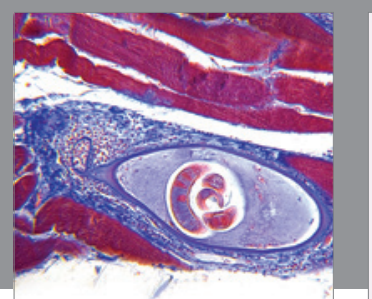

Gastroenterology Research and Practice

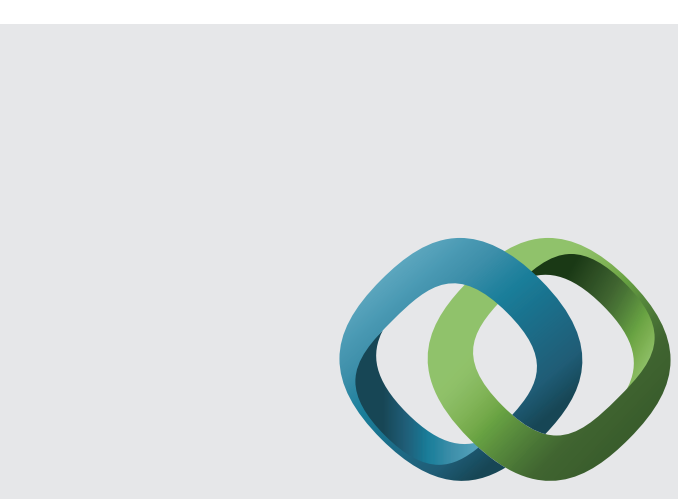

\section{Hindawi}

Submit your manuscripts at

http://www.hindawi.com
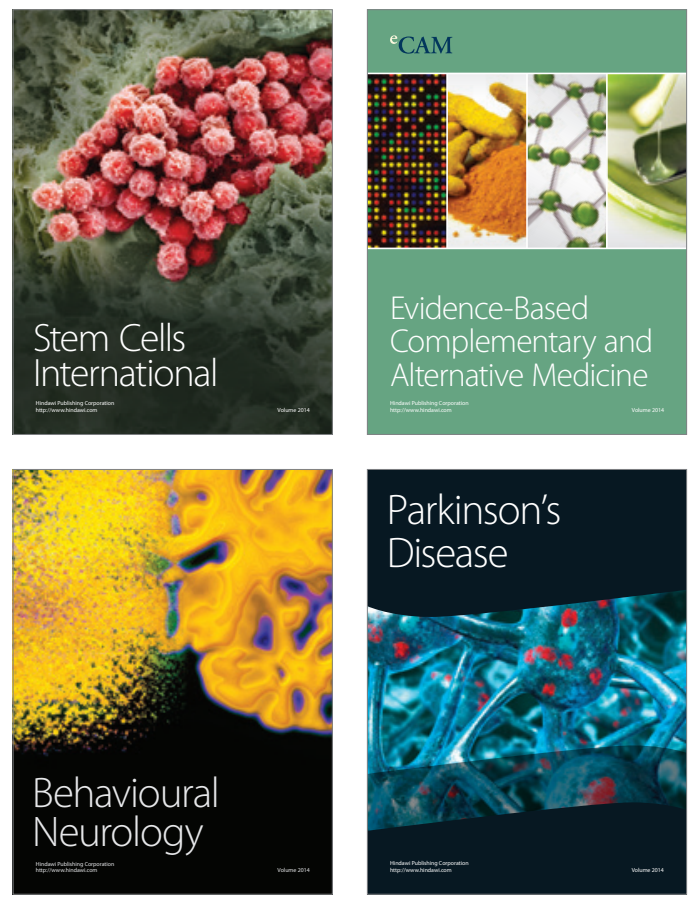
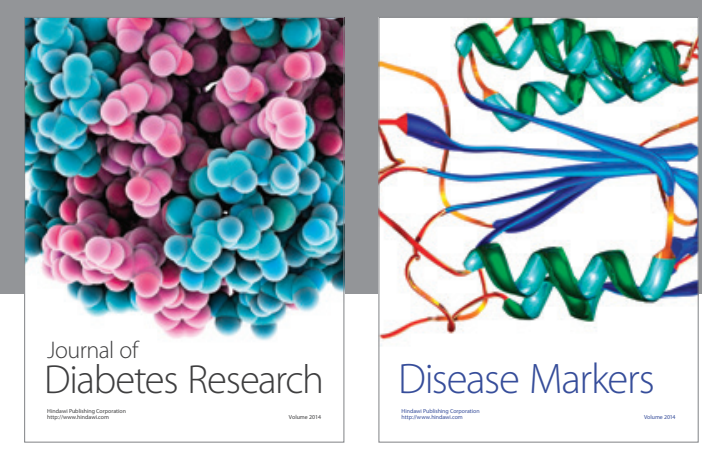

Disease Markers
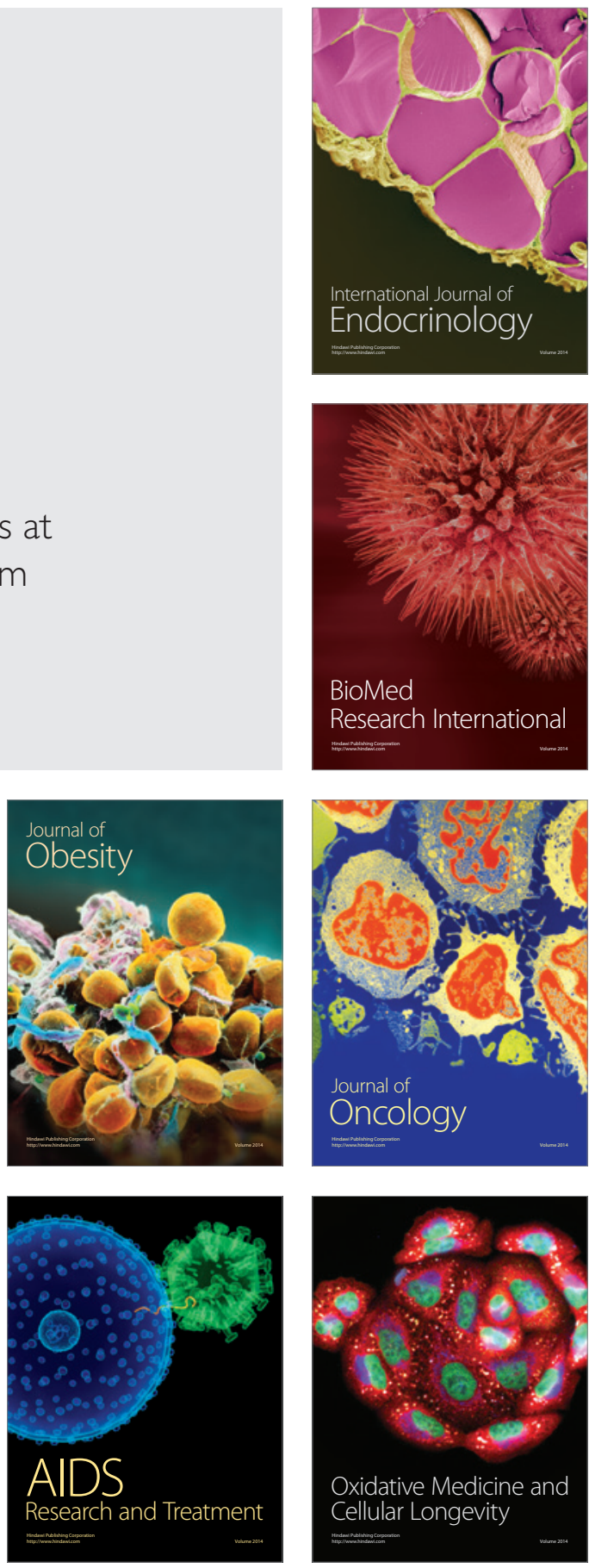